\title{
Imagining Emotional Events Benefits Future-Oriented Decisions
}

\author{
Braedon C. Ballance ${ }^{1}$, Young Ji Tuen ${ }^{1}$, Aria S. Petrucci ${ }^{1}$, William Orwig ${ }^{1}$, Omran K. \\ Safi ${ }^{1}$, Christopher R. Madan ${ }^{2}$, and Daniela J. Palombo*1 \\ ${ }^{1}$ University of British Columbia, Department of Psychology \\ ${ }^{2}$ University of Nottingham, School of Psychology
}

\begin{abstract}
Author Note
Corresponding Author: Daniela J. Palombo. Email: daniela.palombo@ubc.ca; Tel: 604 822 4156. 2136 West Mall, Vancouver, BC V6T $1 Z 4$
\end{abstract}




\begin{abstract}
How does imagining future events - whether positive or negative - influence our choices in the present? Prior work has shown the simulation of hypothetical future events, dubbed episodic future thinking, can alter the propensity to engage in delay discounting (the tendency to devalue future rewards) and does so in a valence specific manner. Some research shows that positive episodic future thinking reduces delay discounting, whereas negative future thinking augments it. However, more recent research indicates that both positive and negative episodic future thinking reduce delay discounting, suggesting an effect of episodic future thinking that is independent of valence (Bulley et al., 2019). In the present study, we sought to replicate and extend these latter findings. Here, participants $(N=604)$ completed an online study. In the baseline task, participants completed a delay discounting task. In the experimental task, they engaged in episodic future thinking before completing a second delay discounting task. Participants were randomly assigned to engage in either positive, neutral, or negative episodic future thinking. In accordance with Bulley et al., we found that episodic future thinking, regardless of valence, reduced delay discounting. Although episodic future thinking shifted decision-making in all conditions, the effect was stronger when participants engaged in positive episodic future thinking, even after accounting for personal relevance and vividness of imagined events. These findings suggest that episodic future thinking may promote future-oriented choices by contextualizing the future, and this effect is further strengthened when the future is tied to positive emotion.
\end{abstract}

Keywords: decision-making, delay discounting, emotion, episodic future thinking, intertemporal choice 


\section{Imagining Emotional Events Benefits Future-Oriented Decisions}

Imagination plays a central role in human cognition. Episodic future thinking refers to one form of imagination wherein one can simulate, in their mind's eye, hypothetical future scenarios that are constructed de novo based on the building blocks of our prior experiences (Schacter \& Addis, 2017). This remarkable human feat - often referred to as mental time travel (Schacter et al., 2017; Suddendorf \& Corballis, 2007; Atance \& O'Neill, 2001; Tulving, 2001; Szpunar, 2010) - occupies a considerable portion of our daily thoughts (D'Argembeau et al., 2010).

Episodic future thinking has become an important topic in the field of behavioural economics, particularly in the context of intertemporal choices, a form of decision making that is ubiquitous in humans. It is well known that humans have a tendency, known as delay discounting, to discount future rewards in favor of immediate ones (Berns et al., 2007; Kirby, 1997). For example, when given a choice between $\$ 70$ now versus $\$ 85$ in two months, individuals tend to choose the smaller, immediate reward. This inclination toward delay discounting demonstrates that individuals will devalue the larger, future reward because of the delay in receiving it (Green \& Myerson, 2004; Kirby, 1997; Mazur, 1987). However, recent empirical work has shown that engaging in episodic future thinking before making choices between immediate and future rewards can attenuate delay discounting (Benoit, Gilbert, \& Burgess, 2011; Bulley et al., 2016; Peters \& Büchel, 2010; Rösch et al., 2021; Rung \& Madden, 2018; also see Schacter et al., 2017; Atance \& O'Neill, 2001). This effect holds regardless of whether the episodic future thinking is related to the reward (Benoit et al., 2011) or a general, unrelated future event (Cheng et al., 2012). Consistent with Pascal Boyer's 
(2008) proposal that episodic future thinking acts as a motivational 'brake' to counter myopic decisions, these findings suggest that episodic future thinking may prompt individuals to assign greater value to future rewards, thereby resisting the urge to opt for immediate gratification (Benoit et al., 2011; Gilbert \& Wilson, 2007; Peters \& Büchel, 2010; Rösch et al., 2021). As detailed below, in this paper, we build on previous work by examining how the emotional valence of episodic future thinking influences delay discounting.

Regarding the relevance of emotional valence to episodic future thinking, Boyer (2008) postulated that episodic future thinking can alter the subjective value of the future by enabling individuals to 'pre-feel' the emotional state of the future. When pre-feeling a positive future experience, that imagined emotional state overrides the pleasure tied to the present reward (also see Benoit et al., 2011; Palombo et al., 2015; Peters \& Büchel, 2010). Thus, when individuals simulate positive future events, episodic future thinking may prompt individuals to invest in the future (Boyer, 2008).

Still, whereas Boyer's proposal focuses on the benefits of positive valence, there are differing viewpoints in the literature on how episodic simulation of negatively valenced future events might influence decision making. According to one view, simulating negative future events biases individuals towards immediate gratification given the unfavorable depiction of the future (Frankenhuis et al., 2016). Indeed, higher levels of worry (often encompassing thoughts about future threat) are associated with steeper delay discounting (Worthy et al., 2014). According to this view, negative episodic future thinking should augment delay discounting. Yet, an alternative viewpoint proposes that negative episodic future thinking may act as a preparatory motivation that 
prompts individuals to invest in the future in an effort to acquire the appropriate resources to manage the future threat (see e.g., Bulley et al., 2016; Worthy et al., 2014; also see Lempert et al., 2012; Bulley et al., 2019). That is, negative episodic future thinking should attenuate delay discounting. Hence, episodic future thinking, irrespective of valence, may adaptively shift decision biases to suit the individual's view of the future (Rösch et al., 2021).

Along these lines, previous research has investigated the manner in which the emotional valence of the episodic future forethought modulates intertemporal choices (Bulley et al., 2019; Liu et al., 2013; Zhang et al., 2018; Calluso et al., 2019). In such studies, participants are prompted to imagine episodic future events before making an intertemporal choice. Whether the participants were prompted to imagine a positively valenced (e.g., winning an award), negatively valenced (e.g., being assaulted by a stranger) or neutral (e.g., using a pencil) event differed between conditions. As expected, participants in such studies discounted the future choice less after engaging in positively valenced episodic future thinking compared to neutral imagination or 'baseline' intertemporal choices with no episodic future thinking component (i.e., attenuated delay discounting; Bulley et al., 2019; Liu et al., 2013; Zhang et al., 2018; Calluso et al., 2019).

However, results from studies where participants engaged in negatively valenced episodic future thinking were equivocal. Two of these studies found that imagining negative future scenarios biased choice towards the smaller, sooner reward (i.e., augmented delay discounting; Liu et al., 2013; Zhang et al., 2018). A major limitation of both these studies was the relatively small samples (approximately 30 
participants per group in between-subjects designs). Contrarily, a third study, using the largest sample size to date $(N=301$, between-subjects design; approximately 100 participants per group), found that, much like positive future scenarios, imagining negative future scenarios biased choice towards the larger, later reward (i.e. the futureoriented reward), albeit the effect size was smaller for negative $(d=.45)$ compared to positive $(d=.63)$ events when each condition was compared to a neutral imagery control condition (Bulley et al., 2019). This pattern of results was also observed in a fourth study by Calluso et al. (2019), with a smaller sample $(N=65$; within-subjects design). Accordingly, a recent meta-analysis which included both published and unpublished data, showed that positive episodic future thinking had a strong effect in reducing delay discounting, whereas negative episodic future thinking had no significant effect (Rösch et al., 2021). Given the inconsistent results, it remains unclear whether both positively and negatively valenced episodic future thinking have a similar (i.e., both reduce delay discounting) or different (i.e., positive reduces delay discounting while negative increases or has no effect on delay discounting) influence on intertemporal choices.

In the present study, we had two primary objectives. Our first objective was to conceptually replicate the findings of Bulley et al. (2019), showing that imagining both positive and negative events attenuate discounting and do so to a greater extent relative to neutral events. Our second objective was to extend these findings by isolating the influence of the emotional valence of episodic future thinking on delay discounting. Consequently, we deviated from the design of Bulley et al. (2019) in a number of ways. First, we compared the effects of both positive and negative episodic future thinking to a 
condition involving neutral episodic future thinking, whereas Bulley et al. (2019) used an atemporal neutral condition (i.e., one that did not involve envisioning the future). For example, a participant in the positive condition might be prompted to imagine 'seeing live music in 6 months' while a participant in the atemporal neutral condition might be prompted to imagine 'leaning on a table'. Second, we asked participants to imagine future neutral events which were more consequential (e.g., attending a work meeting) than those used in prior studies (e.g., folding a piece of paper; also see Liu et al., 2013; Zhang et al., 2018; Bulley et al., 2019). We increased the personal relevance of neutral prompts by having participants generate the prompts themselves, rather than supplying a predetermined list of events - a method which has been used in past work with episodic future thinking (D'Argembeau et al., 2008). In this regard, some of our design features demonstrated parallels those of Calluso et al. (2019), wherein the authors also employed a condition involving personally relevant neutral episodic future thinking.

In line with Bulley et al. (2019), we hypothesized that prompting participants to imagine either positive or negative events would result in a larger shift towards more patient choices (i.e. decreased delay discounting) compared to when participants are prompted to imagine neutral events. If so, these findings would indicate that episodic future thinking promotes future-oriented decisions independent of their emotional valence. Regardless of our findings, this work will help resolve the uncertainty surrounding the effects of negatively valenced episodic future thinking and further reveal how emotionally valenced episodic future thinking influences intertemporal choices.

In addition to our primary objectives, an exploratory goal of this study was to examine whether symptoms of anxiety and depression influence the relationship 
between emotionally valenced episodic future thinking and delay discounting.

Researchers have found that individuals with higher trait anxiety or depression are more likely to imagine negative events when engaging in episodic future thinking (Miloyan et al., 2014; Miloyan \& Suddendorf, 2015; Roepke \& Seligman, 2016) and may display a different trend towards immediate- or future- oriented choices (Bulley et al., 2016). Other work shows that individual differences in stress appraisal interacts with acute stress to influence delay discounting (Lempert et al., 2012). Individuals with higher anxiety and/or depression may be biased towards negative interpretations of the future, even in positive or neutral conditions. To address this goal, the present study also included self-report measures of anxiety and depression so that symptom severity scores could be correlated with the shift in delay discounting due to episodic future thinking. Given the exploratory nature of this analysis, we did not hypothesize the directionality of this relationship.

\section{Methods}

\section{Participants}

A total of 604 participants were recruited through the University of British Columbia's Human Subject Pool and randomly assigned to a positive, neutral, or negative emotional valence condition. As per our pre-registration, we opted to collect responses until we reached $N=650$ or until our sign-up deadline. In determining the sample size for the present study, we did not perform a power analysis, but rather set out to recruit at least as many participants as Bulley et al. $(2019 ; N=297)$. To our knowledge, our study represents the largest sample to date investigating this topic. All participants were fluent in English, provided informed consent, were given the option to 
withdraw from the study at any time, and were compensated in the form of course credit. The protocol for this study was reviewed and approved by the University of British Columbia Behavioural Research Ethics Board.

In accordance with our pre-registration plan, participants were excluded from analysis if they answered three or more (out of five) comprehension questions incorrectly. We also excluded participants if three or more of their imagination prompt descriptions did not describe their thoughts or events and if they did not enter five selfgenerated imagination prompts. For example, a participant would be excluded based upon their imagination event prompts or written descriptions if they skipped through written sections by entering "NA" or nonsensical characters, or copy/pasted their written responses from prior responses. The latter two exclusion criteria were not preregistered. Based on these exclusion criteria, 32 participants were excluded from analysis (5.3\%), leaving a final sample size of 572 participants (Positive $=194$, Neutral $=185$, Negative = 193; see Table 1 for demographics) .

\section{Measures}

\section{Delay Discounting Task}

The intertemporal choice task used in the present study was modified from the 27-item Monetary Choice Questionnaire (MCQ; Kirby et al., 1999) and was used to calculate the proportion of larger, later choices for each participant. The questionnaire was administered twice, once to establish a baseline and a second time in conjunction with episodic future thinking event prompts, subjective ratings (vividness, emotionality, personal relevance), and narrative descriptions, as described in detail below (see 
Procedures). Note that the inclusion of a baseline is a departure from Bulley et al. (2019).

In each item of the modified MCQ, participants were instructed to indicate their preference between a hypothetical reward available immediately (e.g., $\$ 25$ now) or after an indicated delay (e.g., $\$ 60$ after 1, 2, 3, 6, or 12 weeks). The order of intertemporal choices was randomized across trials. See Table 3 for a full list of choices. Using the choices that participants made across different reward values and delays, we calculated the proportion of larger, later choices across the 27 trials for each participant. In this modified version of the questionnaire, monetary values from the original questionnaire were retained but the delays were changed such that there were only five unique delays $(1,2,3,6$ and 12 weeks) instead of a range of delays. Our purpose in modifying these delays was to synchronize the time scales between intertemporal choices and episodic future thinking trials. For example, participants would be prompted to imagine an event that occurs in 6 weeks and then decide between an immediate reward or a reward available in 6 weeks.

\section{Self-Report Measures}

Demographics and Health History. Basic demographic information was assessed with a set of questions regarding age, gender, and education. Participants also completed a health history questionnaire which included questions about drug use, vision and hearing, major health events, and current or past history of neurological and psychiatric concerns. 
State-Trait Anxiety Inventory. To measure symptoms of anxiety, we used the State-Trait Anxiety Inventory (STAI), which consists of two scales measuring state and trait anxiety respectively (Spielberger et al., 1983). State anxiety describes the current level of anxiety (how a participant is feeling at that moment), whereas trait anxiety describes general anxiety (how anxious the participant usually feels). Both scales consist of 20 questions which are rated along a 4-point scale $(1=$ not at all; $4=$ very much so). Anxiety-absent items were reverse coded. Total scores within each subscale range from 20 to 80 , with higher scores indicating greater levels of state or trait anxiety, respectively. Both scales have demonstrated good reliability ( $\alpha=.90$ for STAI-Trait; $\alpha=.92$ for STAI-State; Spielberger et al., 1983).

Centre for Epidemiologic Studies Depression Scale. To measure symptoms of depression, we used the Centre for Epidemiologic Studies Depression Scale (CESD; Radloff, 1977). The CESD consists of 20 items representing various symptoms of depression. Participants rate how often they have experienced each symptom 'during the past week' using a four-point scale $(0=$ not at all or less than 1 day; 3 = most or all of the time, 5-7 days). Depression-absent items were reverse coded. Total scores range from 0 to 60 , with higher scores indicating greater frequency of depressive symptoms. The CESD has shown to be a reliable measure of depression in the general population ( $\alpha=.85$; Radloff, 1977).

\section{Procedure}

In this online study, participants provided informed consent before being randomly assigned to one of three conditions (positive, negative, or neutral future episodic thinking). Participants then completed the two-part delay-discounting task 
followed by self-report measures; see Figure 1 for a visual representation of this task. For the first part of the delay-discounting task, participants completed a baseline iteration of the modified MCQ (question order randomized), which did not involve an episodic future thinking component.

Participants then answered a series of questions regarding their demographics and health history. Next, participants were asked to generate five prompts describing possible future events that they could envision reasonably occurring in their lives within the next four months. By having participants generate these prompts themselves, we aimed to maximize the personal relevance of imagined events across conditions. Specifically, participants were asked to generate specific, novel events (i.e., events they had not already experienced in their past) lasting no longer than 24 hours. Depending on condition, participants generated positive, neutral, or negative future events. To assist with event selection, a list of suitable (and unsuitable) example events was provided for each condition (Table 2). Suitable events were specific and novel, while unsuitable examples were vague and/or familiar.

Participants then completed the second iteration of the modified MCQ (question order randomized). Prior to each trial, participants were randomly prompted to imagine themselves experiencing one of the five self-generated events as vividly as possible while concentrating on details of the experience (e.g., where they are, what they are doing, how they feel); there was no time limit for this. Before beginning this iteration of the modified $M C Q$, participants saw a guided example illustrating the amount of detail they should include when imagining these events. We specified that participants should imagine these events prior to each intertemporal choice question, 
but that responses to those questions did not need to be related to the event. For each trial of the modified MCQ, participants responded to an intertemporal choice question and then rated how vivid $(1=$ not vivid at all; $7=$ very vivid $)$, emotional $(1=$ intensely negative; $4=$ neutral; $7=$ intensely positive $)$, and personally relevant $(1=$ not relevant at all; 7 = very relevant) they considered the event they had imagined just prior to their decision. To affirm that participants were performing the task as instructed, they were asked to provide a written, detailed description of the event they imagined on five randomly selected trials (see Figure 1).

Throughout the survey, participants were asked to answer five, two-option comprehension questions after reading the instructions pertaining to each task. These questions were designed to test whether the participant properly understood the instructions for each task (baseline delay discounting, future event generation and postepisodic future thinking delay discounting). If participants answered this question incorrectly, another prompt would appear summarizing the key points to correct their understanding.

After completing the second iteration of the MCQ, participants completed the STAI and CESD. Participants were then asked to answer questions about the task, including 'What did you think about the study?', 'What did you think the study was about?', and 'Do you have any comments about the study?'. Finally, they were debriefed in full.

\section{Statistical Analysis}


All analyses were conducted using $R$ ( $R$ Core Team, 2019). To confirm that episodic future thinking event prompts elicited the appropriate emotional responses, we conducted a one-way ANOVA to compare emotionality ratings across conditions (positive, negative, or neutral). In addition, we conducted two more one-way ANOVAs to determine whether vividness and personal relevance ratings differed between positive, neutral, and negative conditions.

From both the baseline and imagination delay discounting tasks, we calculated the proportion of larger, later choices to smaller, sooner choices. These proportions were taken as our primary dependent variable and labelled 'LL proportion'. We compared the effect of positive, neutral, or negative episodic future thinking on LL proportions by conducting a $3 \times 2$ mixed ANOVA where the between-subjects factor was emotional valence of episodic future thinking (positive, neutral, or negative) and the within-subjects factor was phase (baseline or post-episodic future thinking delay discounting), as per our pre-registration plan.

Significant interactions were decomposed using post-hoc t-tests. Additionally, we calculated a difference score between delay discounting trials (episodic future thinking LL proportion minus baseline LL proportion) for each participant, which we used in post-hoc analyses to compare the magnitude of effects between conditions (using $t$ tests). Finally, to account for the role of personal relevance and vividness in observed condition differences (which differed across conditions), we conducted two ANCOVAs with personal relevance and vividness as covariates. These latter analyses were not specified in our pre-registration plan. Our a-priori alpha level was set at .05, and 
Bonferroni corrections were used to adjust reported $p$-values for multiple comparisons where stated.

We additionally examined delay discounting using a hyperbolic discounting function. Bulley et al. (2019) used the standard 27-item MCQ (Kirby et al., 1999) and fit $\mathrm{k}$ values based on a hyperbolic discounting function using Kaplan et al.'s (2016) scoring method. As we modified the MCQ to more closely align with our imagined future event methods, we could not use this approach. Here, we used a hierarchical Bayesian estimation method to model hyperbolic discounting parameters for each of the three groups using Vincent's (2016) delay discounting toolbox; model fitting was conducted using Markov-chain Monte-Carlo (MCMC) as implemented in JAGS (Plummer, 2003). This approach involves fitting parameters at the trial, participant, and group levels, somewhat similar to mixed-effect models. In Supplemental Materials, we report the comparison of this approach being applied and the $\mathrm{k}$ values estimated in Bulley et al. (2019). As both methods were relatively consistent and Vincent's (2016) toolbox is more flexible, we applied the latter to our data. This analysis was not pre-registered but was conducted to align our findings with the literature.

Subsequent exploratory analyses were performed to determine whether anxiety and depression symptoms were correlated with shifts in choices between episodic future thinking and baseline delay discounting tasks in each condition, as per our pre-registration. To this end, we computed Pearson and Spearman correlations between the difference scores (episodic future thinking LL proportion minus baseline LL proportion) and STAI-Trait score as well as CESD scores.

\section{Results}


Analyses reported here were in accordance with our pre-registration plan unless otherwise specified (see https://aspredicted.org/ex5gp.pdf).

\section{Summary Statistics}

Table 4 summarizes the primary variables of interest in the present study. These variables include LL proportions (for baseline and post episodic future thinking delay discounting trials), vividness, emotionality, personal relevance ratings, CESD scores and STAI scores. LL proportions and episodic future thinking event ratings were computed by averaging the responses for each participant across the 27 trials of the baseline or episodic future thinking discounting task. CESD and STAI were computed by summing the relevant scores across participants in each condition.

All data from the present study has been made publicly available and can be found at: https://osf.io/m2xv4/?view only=7993664f81344983bc557ae45e0a1b7c.

\section{Episodic Future Thinking Event Ratings}

We first checked whether episodic future thinking event prompts inspired the appropriate emotional response (positive, neutral, or negative) by comparing emotionality ratings across conditions using a one-way ANOVA. As expected, ratings differed across conditions $\left(F(2,569)=625.39, p<.001, \eta p^{2}=.69\right)$ such that participants rated that they felt more positive when imagining events in the positive condition compared to the neutral ( $p<.001$, Cohen's $d=2.29)$ or negative condition $(p<.001, d$ $=3.14$; see Figure 2A). Similarly, participants in the negative condition reported feeling significantly more negative after imagining events compared to participants in the neutral condition $(p<.001, d=1.76$; see Figure $2 \mathrm{~A})$. These results indicated that the 
event prompts used in each of the episodic future thinking discounting trials evoked the expected emotional response in each condition (positive, neutral, or negative).

We next compared vividness ratings across conditions with a one-way ANOVA. We found that vividness ratings differed between conditions $(F(2,569)=7.32$, $\left.p<.001, \eta p^{2}=.03\right)$ such that participants in the positive $(p<.001, d=0.37)$ or negative $(p=.016, d=0.27)$ conditions reported their imaginations to be significantly more vivid than those in the neutral condition (see Figure 2B). However, there was no significant difference in vividness ratings between participants in the positive versus negative condition $(p=1, d=0.10)$. As expected, these findings suggest that participants are more easily able to generate vivid imaginations of positive or negative events (compared to neutral) but there is no difference in the vividness of imaginations between positive and negative events.

Using a third one-way ANOVA, we compared personal relevance ratings across conditions. We found that personal relevance ratings significantly differed between conditions $\left(F(2,569)=39.18, p<.001, \eta p^{2}=.12\right)$ such that participants in the positive condition rated their imaginations as significantly more personally relevant than those in the neutral $(p<.001, d=0.94)$ or negative conditions $(p<.001, d=0.52$; see Figure $2 \mathrm{C}$ ). Additionally, participants in the negative condition also rated their future events as significantly more personally relevant than those in the neutral condition $(p<$ $.001, d=0.39$; See Figure $2 \mathrm{C}$ ). These results indicate that the personal relevance of episodic future thinking differs significantly depending on the emotional valence of the events. 
Given the significant differences in vividness and personal relevance between conditions, we added both variables as covariates in later analyses to determine whether they influenced results.

\section{Effects of Emotionally Valenced Episodic Future Thinking on Delay Discounting}

Our primary objective was to determine whether positive, neutral, or negative episodic future thinking would influence delay discounting differently. To examine this, we conducted a mixed ANOVA with phase (baseline or future thinking) entered as the within-subjects factor and condition (positive, negative, or neutral) entered as the between-subjects factor with LL proportion as the dependent variable. We observed significant main effects of phase $\left(F(1,569)=118.79, p<.001, \eta p^{2}=.17\right)$, but not condition $\left(F(2,569)=1.50, p=.23, \eta p^{2}=.01\right)$ on the LL proportion. Critically, the difference in LL proportion between baseline and episodic future thinking delay discounting depended on condition, as evidenced by a significant phase $\times$ emotional valence interaction $\left(F(2,569)=7.65, p<.001, \eta p^{2}=.03\right)$. Note that the interaction was significant even after controlling for personal significance $(p=.005)$ and vividness $(p=$ .002). To determine the nature of this interaction, we conducted post hoc comparisons with Bonferroni adjustments to correct for multiple comparisons.

We first investigated the effect of condition at each phase (baseline or episodic future thinking) using two one-way ANOVAs. As expected, the effect of condition was significant only in the episodic future thinking condition $\left(F(2,569)=4.26, p=.02, \eta p^{2}=\right.$ .02 ) but not the baseline condition $\left(F(2,569)=.18, p=.84, \eta p^{2}=.001\right)$. Next, we conducted Bonferroni adjusted post hoc comparisons to compare baseline and episodic future thinking delay discounting LL proportions within each level of condition. We found 
that participants in the positive $(p<.001, d=0.32)$, neutral $(p<.001, d=0.76)$ and negative $(p<.001, d=0.35)$ conditions made more larger, later choices in the episodic future thinking delay discounting trials than in the baseline delay discounting trials (Figure 3). These results suggest that engaging in episodic future thinking of any valence (positive, neutral, or negative) promotes larger, later choices.

To break down the above-mentioned interaction between phase and condition, we computed a difference score within each condition and conducted a final set of post hoc comparisons, reporting $p$-values with Bonferroni adjustment. Here, our dependent variable was the difference between episodic future thinking and baseline delay discounting proportions of larger, later choices (a difference score was calculated for each participant). Critically, given the significant differences in vividness and personal relevance ratings between conditions, we ran these analyses using two separate ANCOVAs, where personal relevance and vividness ratings were added as covariates. When controlling for personal relevance, we found that positive episodic future thinking significantly shifted decisions towards larger, later choices compared to neutral $(F(1$, $\left.376)=11.70, p<.001, \eta p^{2}=.03\right)$ and negative $\left(F(1,384)=3.93, p=.048, \eta p^{2}=.01\right)$ episodic future thinking. By contrast, there was no significant difference between the neutral and negative condition $\left(F(1,375)=1.76, p=0.19, \eta p^{2}=.005\right)$. Likewise, when controlling for vividness ratings, we found that positive episodic future thinking significantly shifted decisions towards larger, later choices compared to both neutral $\left(F(1,376)=14.83, p<.001, \eta p^{2}=.04\right)$ and negative $\left(F(1,384)=4.47, p=.035, \eta p^{2}=\right.$ 
.01) episodic future thinking. By contrast, there was no significant difference between the neutral and negative condition $\left(F(1,375)=1.96, p=0.16, \eta p^{2}=.005\right){ }^{1}$

A mixed ANOVA of phase and condition using estimated log $(k)$ values produced similar results to the comparable as the initial ANOVA based on LL proportion. There was a significant main effects of phase $(F(1,569)=141.20, p<.001$, $\left.\eta p^{2}=.20\right)$, but not condition $\left(F(2,569)=2.75, p=.065, \eta p^{2}=.01\right)$. As with LL proportion, there was a significant phase $\times$ emotional valence interaction $(F(2,569)=$ $\left.9.39, p<.001, \eta p^{2}=.03\right)$. Due to these consistencies, we did not repeat the subsequent one-way ANOVAs and separate ANCOVAs again for the $\log (k)$ values. Hyperbolic discounting functions for the groups and phases are shown in Figure 4. Note that this analysis was not pre-registered.

\section{Exploratory analyses}

A peripheral goal was to explore whether higher levels of trait anxiety (measured by the STAI-Trait scale) and depression (measured with the CESD) were correlated with the shift towards larger, later choices after episodic future thinking in each condition. To do so, we calculated the Pearson and Spearman correlation coefficient between STAI-Trait scores and delay discounting difference scores (baseline minus future thinking delay discounting proportion) in the positive, neutral, and negative condition (note that STAI-State, STAI-Trait, and CESD data were missing for $N=4, N=$

\footnotetext{
${ }^{1}$ For completeness, in the Supplementary Materials we also report correlations between the difference score for each condition and ratings of emotionality, vividness, and personal relevance to determine whether the magnitude of the with the shift towards larger, later choices after episodic future thinking was correlated with the phenomenological characteristics of the imagined events. Given the skewness of some ratings, we report both Pearson and Spearman correlations. These analyses were not pre-registered.
} 
2 and $N=2$ participants, respectively). We did not observe any significant correlations between trait anxiety and delay discounting in the positive, neutral, or negative episodic future thinking conditions (all $p$-values $>.27$ ). A similar pattern of results was observed for depression symptoms (all $p$-values > .39). (Note that scores from the STAI-Trait and CESD scales were highly correlated, $r>$.80.)

\section{Discussion}

In the present study, we sought to replicate and extend the work of Bulley et al. (2019), which demonstrated that both positive and negative episodic future thinking attenuated delay discounting. The findings of the present study indicate that engaging in any form of episodic future thinking (whether positive, negative or neutral), promotes patient choices. However, valence did influence this relationship insofar as the magnitude of change in delay discounting (from baseline) was larger for positive episodic future thinking than neutral or negative episodic future thinking; there was no difference in magnitude between negative and neutral episodic future thinking. This pattern held even after controlling for differences in personal relevance and vividness, which covaried with emotion. These findings corroborate past research evidencing the robust ability of positive episodic future thinking to reduce delay discounting (Bulley et al., 2019; Liu et al., 2013; Rösch et al., 2021; Zhang et al., 2018). Critically, the present findings are in line with previous studies (Bulley et al., 2019; Calluso et al., 2019) showing negative episodic future thinking promoted patient choices (i.e., reduced delay discounting), rather than immediate choices (i.e., increased delay discounting) as in prior reports (Liu et al., 2013; Zhang et al., 2018). 
Why does episodic future thinking bias individuals towards patient choices? As discussed by Bulley et al. (2019), one possibility is that episodic future thinking might alter the perception of the future, and thus promote future-oriented choices. This idea is based on Construal Level Theory (Trope \& Liberman, 2010), which posits that temporally distant events are psychologically more distant so individuals construe them more abstractly than immediate events. Along these lines, episodic future thinking prompts individuals to simulate abstract, distant events and represent them more concretely (Cheng et al., 2012; Lempert \& Phelps, 2016; also see Kurth-Nelson et al., 2012). In doing so, individuals may be better equipped to resist the tendency towards delay discounting and instead opt for the future-oriented choice over the immediate one. Such a proposal is consistent with the findings that all episodic future thinking (regardless of emotional valence) shifted participants towards more patient decisions. Although we did not include a group without any episodic future thinking manipulation, other studies have used repeated administrations of a delay discounting task within the same experimental session and demonstrated no change in discounting (e.g., Naudé et al., 2018; Bulley et al, under review; intermixed format). Thus, it is unlikely that participants selected more patient choices in the second iteration of the delay discounting tasks simply due to repeated testing.

Although episodic future thinking promoted greater patience in general, the relatively greater difference score of positive episodic future thinking (compared to neutral or negative) suggests that emotional valence also plays a role. This might occur through a change in construal level, whereby positive episodic future thinking tethers a positive feeling to the future choice. That is, episodic future thinking provides a vehicle 
for value assignment, through which humans can determine if something in the future will feel good or not based on how it makes us feel when we envision it. When such simulations are of positive value, they can most effectively combat the allure of immediate gratification to increase one's fitness goals (Boyer, 2008; Benoit et al., 2011; Palombo et al., 2015; Peters \& Büchel, 2010).

In this light, it is somewhat surprising that negative episodic future thinking also promoted future-oriented choices. From the ideas presented above, it would follow that the construal-level change driven by negative episodic future thinking would reduce the subjective value of the future. Our findings suggest that a different mechanism may come into play when imagined events are negative (also see Bulley et al., 2019; Calluso et al., 2019). Specifically, negative episodic future thinking may compel individuals to select the larger, later reward in an effort to gather resources to combat the effects of an otherwise bleak future (i.e., preparatory motivation; Bulley et al., 2019). This is in line with the idea that the emotional content of episodic future thinking can act as an adaptive motivator to sway present behaviour in whatever direction provides the most biological value (Miloyan \& Suddendorf, 2015). Still, it is important to consider that the effect of negative episodic future thinking was statistically comparable to neutral episodic future thinking in our study. As such, if preparatory motivation is involved in promoting future-oriented choices, it may work in conjunction with a construal-level change driven by episodic future thinking in general.

Although our results are in accordance with both Bulley et al. (2019) and Calluso et al., (2019), it remains a puzzle why some studies have observed an opposite pattern of results: that negative episodic future thinking augments temporal discounting 
(e.g., Liu et al., 2013; Zhang et al. 2018). A content analysis of the types of events produced in studies that observe increased versus decreased temporal discounting following negative episodic future thinking could illuminate whether certain types of negative events are more likely to promote patience. Some types of negative simulations, such as those pertaining to illness or death, may be especially potent in pulling one towards more myopic choice behaviour (Bulley et al., 2016). When episodic future thinking increases the salience of one's shortened time horizon or risk of mortality, delaying gratification becomes a less sensible choice. Related to this idea, the degree of controllability of the negative event may play a role; events with a more malleable outcome may be more effective in promoting farsighted behaviour, as doing so could attenuate the degree to which the future yields harm (see e.g., Bulley et al., 2019; Bulley \& Schacter, 2020 for discussions of this idea). In considering alternative interpretations for these incongruous results, it is possible that other study design features are at play, including sample size, which was notably smaller in Liu et al., (2013) and Zhang et al., (2018) relative to the other studies.

In the present study, we developed a neutral condition that involves engaging in episodic future thinking tied to more meaningful events as opposed to the imagery tasks used in prior studies, which involved envisioning more menial tasks like 'sitting on a chair'. Although we were unsuccessful in fully matching personal relevance between conditions, which was higher for emotional versus neutral events, we note that it may be difficult to do so as high personal relevance may be part and parcel of emotional events. Nevertheless, differences in personal relevance could not explain any effects we observed here; the significant difference between positive and neutral episodic future 
thinking (as well as positive versus negative episodic future thinking) were observed after controlling for personal relevance. This point is relevant in comparing our results to Bulley et al. (2019). In their study, negative episodic future reduced delay discounting in comparison to a neutral imagery control condition; whereas in our study it did so in comparison to a baseline version of the task, but not in comparison to neutral episodic future thinking. Hence, it is important to consider results from studies in light of the control tasks used.

In our exploratory analyses, we found no significant correlation between trait anxiety (or depression) levels and the effect of episodic future thinking on intertemporal choices in any condition. Previous research has shown that individuals with trait anxiety and depression are more prone to imagine negative events when engaging in episodic future thinking (Miloyan et al., 2014; Miloyan \& Suddendorf, 2015; Roepke \& Seligman, 2016). Given this negative bias, researchers have suggested that individuals who display higher levels of trait anxiety or depression might be more prone to selecting immediate intertemporal choices after engaging in episodic future thinking (see Bulley et al., 2016). Yet, our finding that negative episodic future thinking promotes patient choices suggests that even individuals prone to envisioning negative future events would shift their decisions towards future-oriented choices. In any case, our data suggest that the relationship between episodic future thinking and intertemporal choices is not affected by trait anxiety. A caveat is that the present study used a general (not clinical) undergraduate population and as such, further research is needed to definitively determine whether such relationships would manifest in those with clinical levels of trait anxiety (or depression). 


\section{Limitations and Future Directions}

The present study has limitations that merit consideration. First, unlike previous research in this area, the present study was administered online rather than inperson. As such, the present study lacked experimenter control over the environment in which the experiment took place. Although participants who expressed deviating from task instructions were excluded (see Methods), it is possible that some participants deviated from instructions but did not report doing so. If anything, these deviations would introduce noise into our paradigm, and we nevertheless observed significant effects in the present study. Moreover, we note that a recent meta-analysis found no difference in effect sizes between online and in-person studies using episodic future thinking (Rösch et al., 2021).

A second limitation is the possible presence of demand characteristics which could have driven the effect of episodic future thinking on intertemporal choices. Given that episodic future thinking involves shifting participants' focus towards the future, it is possible that participants could deduce the purpose of this manipulation and alter their decisions accordingly. However, researchers have found that the effects of episodic future thinking on delay discounting are not significantly different between those who could correctly identify the hypothesis versus those who could not (Rung \& Madden, 2019). Furthermore, a recent meta-analysis found that the effect of episodic future thinking on delay discounting was the greatest in studies that were less prone towards demand characteristics (Rösch et al., 2021). In the present study, we observed little evidence for the role of demand characteristics, namely that participants were able to 
guess the correct study hypothesis (see Table S4). Thus, it seems that the effect of episodic future thinking on delay discounting is not driven by demand characteristics.

A final limitation of this study is that we did not distinguish between valence and arousal, and future research should consider the extent to which arousal may contribute to the effects observed here. Both subjective (i.e., self report) and objective (i.e., skin conductance) measures could be considered for examining this further (see Greening et al., 2021).

\section{Conclusions}

Episodic simulation allows humans to play out the future and optimize intertemporal choices - a ubiquitous form of decision making in humans (Bulley \& Schacter, 2020). The present findings demonstrate that episodic future thinking, regardless of the emotional valence of simulated content, promotes patient choices and that imagining positive events enhances this effect. These results thus replicate prior work and bring into sharper focus the adaptive value of episodic future thinking. In conclusion, uncovering the mechanisms which underlie the effects of episodic future thinking on intertemporal choices provide useful insight into how the domains of imagination and decision-making interact and manifest in human behaviour. 


\section{References}

Atance, C. M. \& O'Neill, D. K. (2001). Episodic future thinking. Trends in Cognitive Sciences, 5(12):533-539. https://doi.org/10.1016/S1364-6613(00)01804-0

Benoit, R. G., Gilbert, S. J., \& Burgess, P. W. (2011). A Neural Mechanism Mediating the Impact of Episodic Prospection on Farsighted Decisions. Journal of Neuroscience, 31(18), 6771-6779. https://doi.org/10.1523/JNEUROSCI.655910.2011

Berns, G. S., Laibson, D., \& Loewenstein, G. (2007). Intertemporal choice - toward an integrative framework. Trends in Cognitive Sciences, 11(11), 482-488. https://doi.org/10.1016/j.tics.2007.08.011

Boyer, P. (2008). Evolutionary economics of mental time travel? Trends in Cognitive Sciences, 12(6), 219-224. https://doi.org/10.1016/j.tics.2008.03.003

Bulley, A., Henry, J., \& Suddendorf, T. (2016). Prospection and the present moment: The role of episodic foresight in intertemporal choices between immediate and delayed rewards. Review of General Psychology, 20(1), 29-47. https://doi.org/10.1037/gpr0000061

Bulley, A., Miloyan, B., Pepper, G. V., Gullo, M. J., Henry, J. D., \& Suddendorf, T. (2019). Cuing both positive and negative episodic foresight reduces delay discounting but does not affect risk-taking. Quarterly Journal of Experimental Psychology, 72(8), 1998-2017. https://doi.org/10.1177/1747021818819777

Bulley, A., Schacter, D.L. Deliberating trade-offs with the future. Nature Human Behaviour, 4, 238-247 (2020). https://doi.org/10.1038/s41562-020-0834-9

Calluso, C., Tosoni, A., Cannito, L., \& Committeri, G. (2019). Concreteness and emotional valence of episodic future thinking (EFT) independently affect the dynamics of intertemporal decisions. PloS one, 14(5), e0217224. https://doi.org/10.1371/journal.pone.0217224

Cheng, Y. Y., Shein, P. P., \& Chiou, W. Bin. (2012). Escaping the impulse to immediate gratification: The prospect concept promotes a future-oriented mindset, prompting an inclination towards delayed gratification. British Journal of Psychology, 103(1), 129-141. https://doi.org/10.1111/j.2044-8295.2011.02067.x

D'Argembeau, A., Renaud, O., \& Van der Linden, M. (2010). Frequency, characteristics, and functions of future-oriented thoughts in daily life. Applied Cognitive Psychology, 35, 96-103.

D’Argembeau, A., Xue, G., Lu, Z. L., Van der Linden, M., \& Bechara, A. (2008). Neural correlates of envisioning emotional events in the near and far future. Neurolmage, 40(1), 398-407. https://doi.org/10.1016/j.neuroimage.2007.11.025

Frankenhuis, W. E., Panchanathan, K., \& Nettle, D. (2016). Cognition in harsh and unpredictable environments. Current Opinion in Psychology, 7, 76-80. https://doi.org/10.1016/j.copsyc.2015.08.011

Gilbert, D. T., \& Wilson, T. D. (2007). Prospection: experiencing the future. Science (New York, N.Y.), 317(5843), 1351-1354. https://doi.org/10.1126/science.1144161

Green, L., \& Myerson, J. (2004). A discounting framework for choice with delayed and probabilistic rewards. Psychological Bulletin, 130(5), 769-792. https://doi.org/10.1037/0033-2909.130.5.769 
Greening, S.G., Lee, T., Grégoire, L., Burleigh, L., Robinson, T., Jiang, X., Mather, M., Kaplan, J. (2021). Fear in the mind's eye: Mental imagery can generate and regulate acquired differential fear conditioned reactivitybioRxiv https://doi.org/10.1101/2021.02.04.429795

Kirby, K. N. (1997). Bidding on the Future: Evidence Against Normative Discounting of Delayed Rewards. Journal of Experimental Psychology: General, 126(1), 54-70. https://doi.org/10.1037/0096-3445.126.1.54

Kirby, K. N., Petry, N. M., \& Bickel, W. K. (1999). Heroin and cocaine abusers have higher discount rates for delayed rewards than alcoholics or non-drug-using controls. Journal of Experimental Psychology: General, 127, 78-87. https://doi.org/10.1111/j.1360-0443.2003.00669.x

Kurth-Nelson, Z., Bickel, W., \& Redish, A. D. (2012). A theoretical account of cognitive effects in delay discounting. The European Journal of Neuroscience, 35(7), 1052-1064. https://doi.org/10.1111/j.1460-9568.2012.08058.x

Lempert, K. M., \& Phelps, E. A. (2016). The Malleability of Intertemporal Choice. Trends in Cognitive Sciences, 20(1), 64-74. https://doi.org/10.1016/j.tics.2015.09.005

Lempert, K. M., Porcelli, A. J., Delgado, M. R., \& Tricomi, E. (2012). Individual differences in delay discounting under acute stress: the role of trait perceived stress. Frontiers in Psychology, 3, 251. https://doi.org/10.3389/fpsyg.2012.00251

Liu, L., Feng, T., Chen, J., \& Li, H. (2013). The value of emotion: How does episodic prospection modulate delay discounting? PLOS ONE, 8(11). https://doi.org/10.1371/journal.pone.0081717

Mazur, J. E. (1987). An adjusting procedure for studying delayed reinforcement. In Commons, M. L., Mazur, J. E., Nevin, J. A., \& Rachlin, H., editors, Quantitative analyses of behavior: The effect of delay and of intervening events on reinforcement value., volume 5, pages 55-73. Lawrence Erlbaum Associates, Inc. Place: Hillsdale, NJ, US Publisher: Lawrence Erlbaum Associates, Inc.

Miloyan, B., Pachana, N. A., \& Suddendorf, T. (2014). The future is here: A review of foresight systems in anxiety and depression. Cognition and Emotion, 28(5), 795810. https://doi.org/10.1080/02699931.2013.863179

Miloyan, B., \& Suddendorf, T. (2015). Feelings of the future. Trends in Cognitive Sciences, 19(4), 196-200. https://doi.org/10.1016/j.tics.2015.01.008

Naudé, G. P., Kaplan, B. A., Reed, D. D., Henley, A. J., \& DiGennaro Reed, F. D. (2018). Temporal framing and the hidden-zero effect: rate-dependent outcomes on delay discounting. Journal of the experimental analysis of behavior, 109(3), 506-519. https://doi.org/10.1002/jeab.328

Palombo, D. J., Keane, M. M., \& Verfaellie, M. (2015). The medial temporal lobes are critical for reward-based decision-making under conditions that promote episodic future thinking. Hippocampus, 25(3), 345-353. https://doi.org/10.1002/hipo.22376

Peters, J., \& Büchel, C. (2010). Episodic Future Thinking Reduces Reward Delay Discounting through an Enhancement of Prefrontal-Mediotemporal Interactions. Neuron, 66(1), 138-148. https://doi.org/10.1016/j.neuron.2010.03.026 Plummer, M. (2003). JAGS: A program for analysis of Bayesian graphical models using Gibbs sampling. In Proceedings of the 3rd International Workshop on Distributed Statistical Computing (DSC 2003) (pp. 20-22). 
Qualtrics. (2005). Qualtrics (Version November 2020). Qualtrics. https://www.qualtrics.com

R Core Team (2019). R: A language and environment for statistical computing. $R$ Foundation for Statistical Computing, Vienna, Austria. URL https://www.Rproject.org/.

Radloff, L. S. (1977). The CES-D Scale: A Self-Report Depression Scale for Research in the General Population. Applied Psychological Measurement, 1(3), 385-401. https://doi.org/10.1177/014662167700100306

Roepke, A. M., \& Seligman, M. E. P. (2016). Depression and prospection. British Journal of Clinical Psychology, 55(1), 23-48. https://doi.org/10.1111/bjc.12087

Rösch, S. A., Stramaccia, D. F., \& Benoit, R. G. (2021). Promoting Farsighted Decisions via Episodic Future Thinking: A Meta-analysis. PsyArXiv, 1-16. Retrieved from 10.31234/osf.io/53ju2

Rung, J. M., \& Madden, G. J. (2018). Experimental reductions of delay discounting and impulsive choice: A systematic review and meta-analysis. Journal ofExperimental Psychology. General, 147(9), 1349-1381. https://doi.org/10.1037/xge0000462

Rung, J. M., \& Madden, G. J. (2019). Demand characteristics in episodic future thinking II: The role of cues and cue content in changing delay discounting. Experimental and Clinical Psychopharmacology, 27(5), 482-495. https://doi.org/10.1037/pha0000260

Schacter, D. L., \& Addis, D. R. (2007). The cognitive neuroscience of constructive memory: remembering the past and imagining the future. Philosophical transactions of the Royal Society of London. Series B, Biological sciences, 362(1481), 773-786. https://doi.org/10.1098/rstb.2007.2087

Schacter, D. L., Benoit, R. G., \& Szpunar, K. K. (2017). Episodic future thinking: mechanisms and functions. Current Opinion in Behavioral Sciences, 17, 41-50. https://doi.org/10.1016/j.cobeha.2017.06.002

Spielberger, C. D., Gorusch, R. L., \& Lushene, R. E. (1970). State-Trait Anxiety Inventory. In Manual for the State-Trait Anxiety Inventory (pp. 3-4). https://doi.org/10.4135/9781483365817.n1316

Suddendorf, T., \& Corballis, M. C. (2007). The evolution of foresight: What is mental time travel, and is it unique to humans? Behavioral and Brain Sciences, 30(3), 299-351. https://doi.org/10.1017/S0140525X07001975

Szpunar K.K. (2010). Episodic future thought: An emerging concept. Perspectives on Psychological Science, 5:142-162. https://doi.org/10.1177/1745691610362350

Trope, Y., \& Liberman, N. (2010). Construal-Level Theory of Psychological Distance. Psychological Review, 117(2), 440-463. https://doi.org/10.1037/a0018963

Worthy, D. A., Byrne, K. A., \& Fields, S. (2014). Effects of emotion on prospection during decision-making. Frontiers in Psychology, 5, 591. https://doi.org/10.3389/fpsyg.2014.00591

Tulving, E. (2001) Origin of autonoesis in episodic memory. In The Nature of Remembering: Essays in Honor of Robert G. Crowder (Roediger, H.L.I. and Nairne, J., eds), pp. 17-34, American Psychological Association. Vincent, B. T. (2016). Hierarchical Bayesian estimation and hypothesis testing for delay discounting tasks. Behavior Research Methods, 48, 1608-1620. https://doi.org/10.3758/s13428-015-0672-2 
Zhang, S., Peng, J., Qin, L. L., Suo, T., \& Feng, T. (2018). Prospective emotion enables episodic prospection to shift time preference. British Journal of Psychology, 109(3), 487-499. https://doi.org/10.1111/bjop.12284 


\section{Acknowledgements}

This work is supported by an Insight Development Grant awarded to D.J.P. from SSHRC. D.J.P. is also supported by an Natural Sciences and Engineering Research Council of Canada Discovery Grant and a Canadian Foundation for Innovation John R.

Evans Leaders Fund grant. B.C.B., Y.T., and D.J.P. designed the study, all co-authors analyzed the data, B.C.B. wrote the paper with input from all co-authors. The authors thank Kate Checknita and Leor Elizur for research assistance, and Dr. James Enns for his guidance and feedback during early revisions. The authors have no conflicts of interest to declare that are relevant to the content of this article. 


\section{Table 1}

Gender Identities in Positive, Neutral and Negative Conditions

\begin{tabular}{lccc}
\hline & $\begin{array}{c}\text { Neutral } \\
(\mathrm{N}=185)\end{array}$ & $\begin{array}{c}\text { Positive } \\
(\mathrm{N}=194)\end{array}$ & $\begin{array}{c}\text { Negative } \\
(\mathrm{N}=193)\end{array}$ \\
\hline Age & & & \\
Mean (SD) & $20.4(2.30)$ & $20.7(2.75)$ & $20.7(2.52)$ \\
Gender & & & \\
Cisgender & $91(49.2 \%)$ & $70(36.1 \%)$ & $74(38.3 \%)$ \\
Transgender & $1(.5 \%)$ & $0(0 \%)$ & $0(0 \%)$ \\
Woman & $78(42.2 \%)$ & $100(51.5 \%)$ & $96(49.7 \%)$ \\
Man & $13(7.0 \%)$ & $22(11.3 \%)$ & $16(8.3 \%)$ \\
Nonbinary & $0(0 \%)$ & $0(0 \%)$ & $3(1.6 \%)$ \\
Two-Spirit & $0(0 \%)$ & $0(0 \%)$ & $0(0 \%)$ \\
Self-Described & $0(0 \%)$ & $1(.5 \%)$ & $0(0 \%)$ \\
No Answer & $2(1.1 \%)$ & $1(.5 \%)$ & $4(2.1 \%)$ \\
\hline
\end{tabular}




\section{Table 2}

Example Future Event Lists Provided in Positive, Neutral and Negative Conditions

\begin{tabular}{lll}
\hline \multicolumn{1}{c}{ Positive Future Events } & \multicolumn{1}{c}{ Neutral Future Events } & \multicolumn{1}{c}{ Negative Future Events } \\
\hline $\begin{array}{l}\text { Winning a scholarship that } \\
\text { I applied for. }\end{array}$ & $\begin{array}{l}\text { Printing off my completed } \\
\text { assignment. }\end{array}$ & $\begin{array}{l}\text { Falling ill with food } \\
\text { poisoning. }\end{array}$ \\
$\begin{array}{l}\text { Going to see my favourite } \\
\text { musician. }\end{array}$ & $\begin{array}{l}\text { Attending a meeting at } \\
\text { work. } \\
\text { Packing my bag with } \\
\text { everything I need for the } \\
\text { day. }\end{array}$ & $\begin{array}{l}\text { Falling and injuring my } \\
\text { back. }\end{array}$ \\
$\begin{array}{lll}\text { Getting into a traffic } \\
\text { accident. }\end{array}$ \\
$\begin{array}{l}\text { Relaxing at the beach in loved ones. } \\
\text { my dream vacation spot. }\end{array}$ & $\begin{array}{l}\text { Peer-reviewing an in-class } \\
\text { presentation. }\end{array}$ & Contracting an infection. \\
\hline
\end{tabular}

Note. Not included in this table, we provided an equal number of 'bad examples. These 'bad examples' were vague events (i.e., 'having a bad day') that would not be conducive to rich imagination. The table provides four representative examples, but participants were provided with a more exhaustive list. 
Table 3

Intertemporal Choices, including Rewards and Delays

\begin{tabular}{|c|c|c|c|}
\hline $\begin{array}{c}\text { Immediate Reward } \\
(\$)\end{array}$ & $\begin{array}{c}\text { Delayed Reward } \\
\text { (\$) }\end{array}$ & $\begin{array}{l}\text { Delay (in } \\
\text { weeks) }\end{array}$ & $\begin{array}{l}\text { LL Proportion at } \\
\text { Baseline }\end{array}$ \\
\hline 20 & 55 & 1 & 0.99 \\
\hline 31 & 85 & 1 & 0.98 \\
\hline 11 & 30 & 2 & 0.96 \\
\hline 15 & 35 & 2 & 0.95 \\
\hline 33 & 80 & 2 & 0.98 \\
\hline 25 & 60 & 3 & 0.93 \\
\hline 14 & 25 & 3 & 0.71 \\
\hline 41 & 75 & 3 & 0.88 \\
\hline 27 & 50 & 3 & 0.85 \\
\hline 24 & 35 & 3 & 0.60 \\
\hline 54 & 80 & 3 & 0.84 \\
\hline 34 & 50 & 6 & 0.60 \\
\hline 19 & 25 & 6 & 0.35 \\
\hline 55 & 75 & 6 & 0.60 \\
\hline 40 & 55 & 6 & 0.48 \\
\hline 25 & 30 & 6 & 0.24 \\
\hline 49 & 60 & 6 & 0.48 \\
\hline 69 & 85 & 6 & 0.55 \\
\hline 54 & 60 & 12 & 0.19 \\
\hline 54 & 55 & 12 & 0.07 \\
\hline 67 & 75 & 12 & 0.29 \\
\hline 22 & 25 & 12 & 0.11 \\
\hline 80 & 85 & 12 & 0.12 \\
\hline 47 & 50 & 12 & 0.12 \\
\hline 78 & 80 & 12 & 0.10 \\
\hline
\end{tabular}




\begin{tabular}{|l|l|l|l|}
\hline 28 & 30 & 12 & 0.10 \\
\hline 34 & 35 & 12 & 0.06 \\
\hline
\end{tabular}

Note. LL= larger later 


\section{Table 4}

Summary Statistics in Positive, Neutral and Negative Conditions

\begin{tabular}{lccc}
\hline & Neutral & Positive & Negative \\
& $(N=185)$ & $(N=194)$ & $(N=193)$ \\
\hline Baseline DD & & & \\
Mean $(S D)$ & $.52(.21)$ & $.52(.21)$ & $.53(.22)$ \\
Median [Min, Max] & $.52[.07,1]$ & $.48[.04,1]$ & $.52[.04,1]$ \\
EFT DD & & & \\
Mean (SD) & $.56(.21)$ & $.63(.22)$ & $.60(.25)$ \\
$\quad$ Median [Min, Max] & $.56[.11,1]$ & $.63[.07,1]$ & $.63[0,1]$ \\
Emotionality & & & \\
Mean (SD) & $4.15(.51)$ & $5.66(.77)$ & $2.56(1.16)$ \\
Median [Min, Max] & $4.07[1.07,6.96]$ & $5.67[3.19,7]$ & $2.22[1,6.26]$ \\
Vividness & & & \\
Mean (SD) & $5.05(1.13)$ & $5.43(.91)$ & $5.34(.96)$ \\
Median [Min, Max] & $5.11[1,7]$ & $5.48[1.96,7]$ & $5.52[2.37,7]$ \\
Personal Relevance & & & \\
Mean (SD) & $4.90(1.14)$ & $5.83(.82)$ & $5.33(1.08)$ \\
Median [Min, Max] & $4.89[1.07,7]$ & $5.96[2.78,7]$ & $5.44[1.67,7]$ \\
STAI-Trait & & & \\
Mean (SD) & $45.94(11.19)$ & $46.45(11.91)$ & $47.18(11.02)$ \\
Median [Min, Max] & $45[21,73]$ & $47[23,75]$ & $47[22,72]$ \\
Missing & 0 & 2 & 0 \\
CESD & & & \\
Mean (SD) & $19.01(11.58)$ & $19.27(11.16)$ & $20.33(10.97)$ \\
Median [Min, Max] & $17[1,50]$ & $17[1,52]$ & $20[0,47]$ \\
Missing & 1 & 1 & 0 \\
\hline
\end{tabular}

Note. Baseline and episodic future thinking (EFT) variables represent the proportion of larger, later choices in either baseline or EFT delay discounting tasks respectively. 


\section{Figure 1}

Episodic Future Thinking Delay Discounting Trial Design

Generate 5 Future Events (Positive, Neutral or Negative)

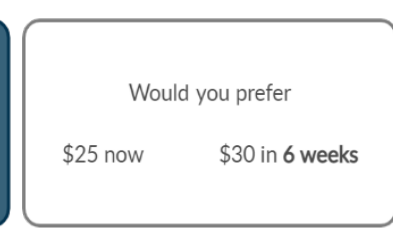

Rate the event you just imagined

(Vividness, Emotionality, Personal Relevance)
Written

Note. This figure depicts the order of events in a single trial of the episodic future thinking delay discounting task. Each participant completed 27 of these trials presented in a randomized order, where one of the five self-generated events was input into each trial. 
Figure 2

Average Emotionality, Vividness, and Personal Relevance Ratings of Episodic Future Thinking Events in Each Condition
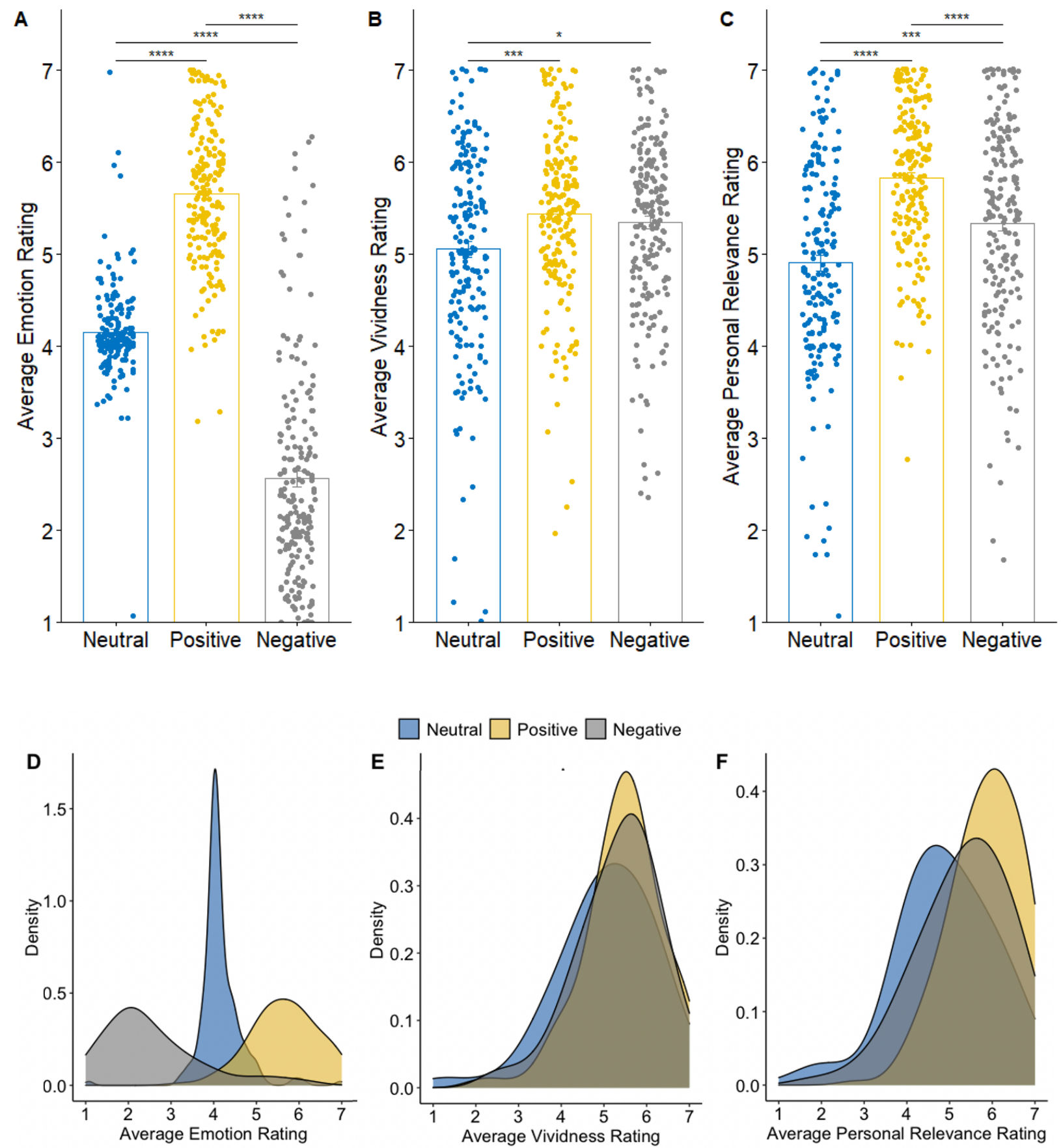
Note. This figure displays the average emotionality $(A)$, vividness $(B)$ and personal relevance $(C)$ ratings from the episodic future thinking delay discounting trials. Bars indicate the mean $( \pm S E)$ of each rating for neutral, positive, or negative conditions. Individual data points for each participant are jittered. D-F. Density plots for A-C. 


\section{Figure 3}

Comparing Baseline and Episodic Future Thinking Delay Discounting Trials in Each Condition

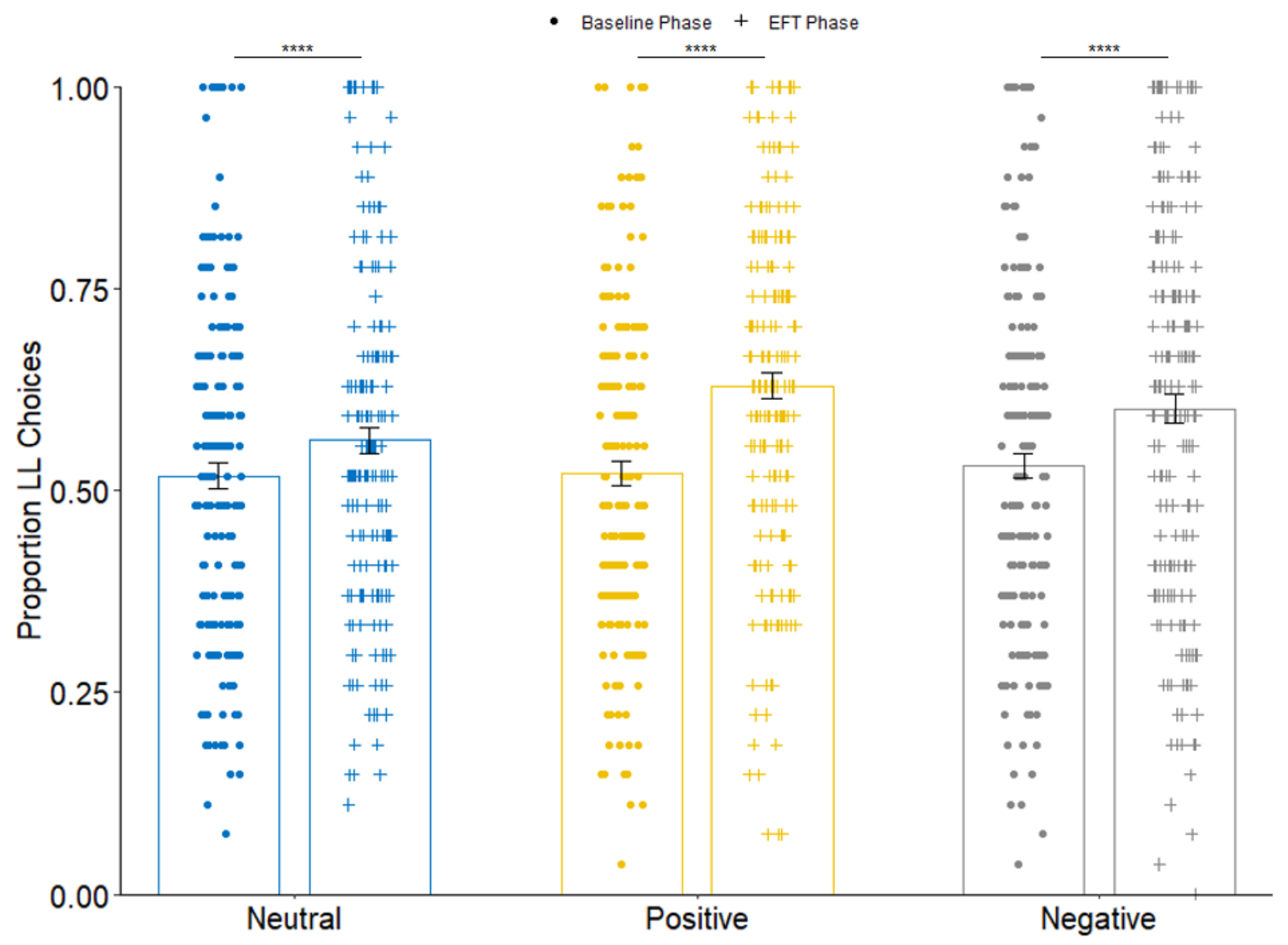

Note. This figure displays the proportion of larger, later (LL) choices in baseline (circles) or episodic future thinking (EFT; plus symbols) for the positive, neutral or negative conditions. Bars indicate the mean ( \pm SE) proportion LL choices for baseline or EFT delay discounting trials in the neutral, positive, and negative conditions. Individual data points for each participant are jittered. 


\section{Figure 4}

Comparing Hyperbolic Discounting Functions for Baseline and Episodic Future Thinking Delay Discounting in Each Condition

A

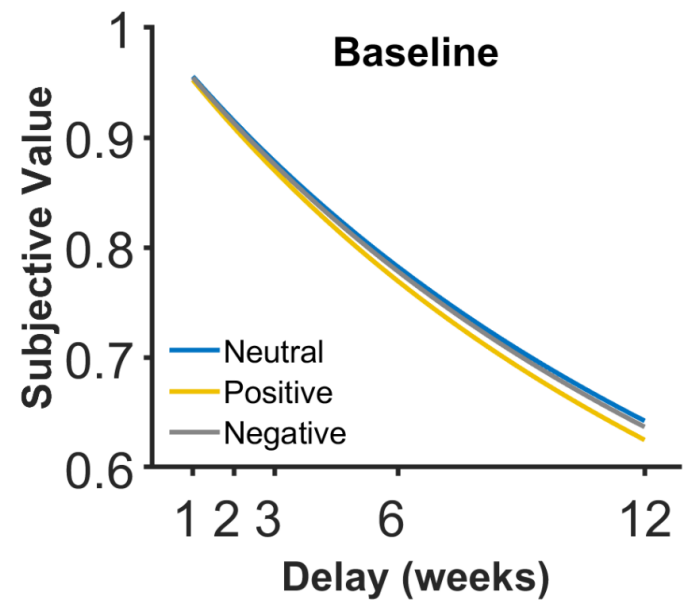

C

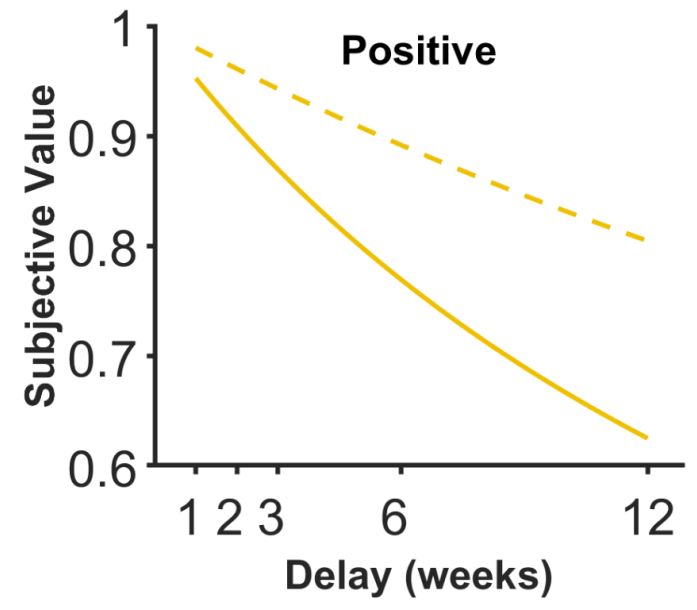

B

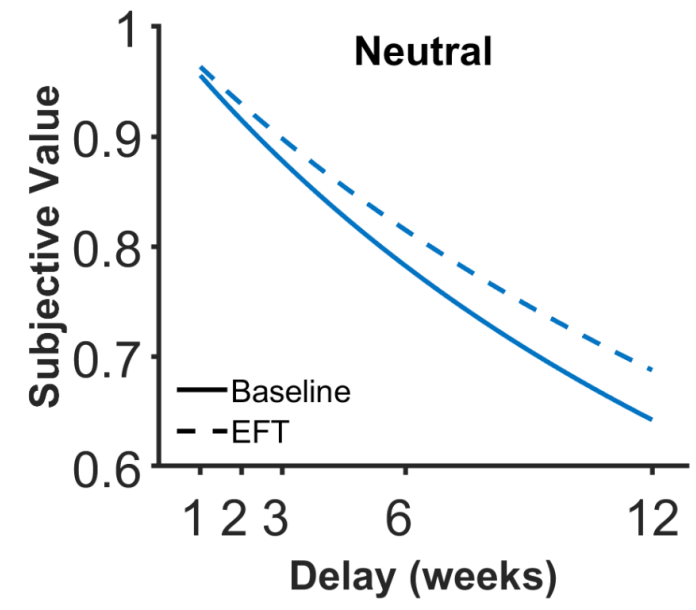

D

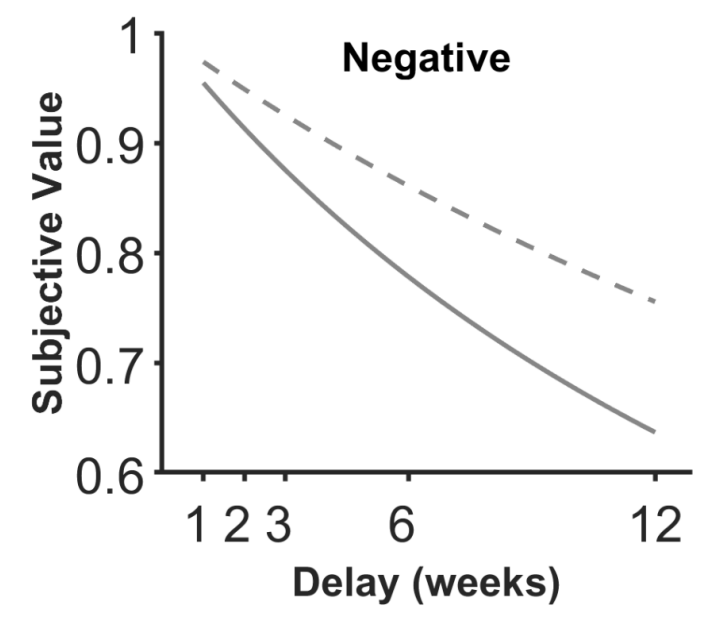

Note. This figure displays the estimated hyperbolic discounting function for baseline or episodic future thinking (EFT) delay discounting trials for positive, neutral or negative conditions. Panel A shows the baseline discounting functions as being nearly identical for all three groups. Panels B-D show the baseline (solid line) and EFT (dashed line). Note that the shallower discounting overall in our paradigm is likely related to the shorter delays chosen in our study; our maximum delay was 84 days (12 weeks), 
whereas the 27-item MCQ has a maximum delay of 189 days (11 of 27 questions in the MCQ have delays longer than 84 days). 


\section{Supplemental Materials}

\section{Correlations between Delay Discounting Difference Scores and Ratings}

\section{Table S1}

Correlations Between Emotion Ratings and Difference Scores in Each Condition

\begin{tabular}{lccccc}
\hline \multicolumn{5}{c}{ Emotion Ratings - Difference Scores } \\
\hline Condition & $N$ & Pearson & $p$ & Spearman & $p$ \\
\hline Neutral & 185 & .03 & .72 & .09 & .24 \\
Positive & 194 & .15 & .035 & .19 & .009 \\
Negative & 193 & .004 & .95 & .02 & .77 \\
\hline
\end{tabular}

Note. Reported $p$-values are uncorrected.

\section{Table S2}

Correlations Between Vividness Ratings and Difference Scores in Each Condition

$$
\text { Vividness Ratings - Difference Scores }
$$

\begin{tabular}{lccccc}
\hline Condition & $N$ & Pearson & $p$ & Spearman & $p$ \\
\hline Neutral & 185 & .12 & .10 & .22 & .003 \\
Positive & 194 & .23 & .001 & .22 & .002 \\
Negative & 193 & .04 & .63 & .01 & .88 \\
\hline
\end{tabular}

Note. Reported $p$-values are uncorrected. 


\section{Table S3}

Correlations Between Personal Relevance Ratings and Difference Scores in Each Condition

Personal Relevance Ratings - Difference Scores

\begin{tabular}{lccccc}
\hline Condition & $N$ & Pearson & $p$ & Spearman & $p$ \\
\hline Neutral & 185 & .09 & .22 & .16 & .03 \\
Positive & 194 & .06 & .37 & .07 & .33 \\
Negative & 193 & .004 & .95 & .03 & .68 \\
\hline
\end{tabular}

Note. Reported $p$-values are uncorrected.

\section{Scoring of Debrief Responses}

Using a similar approach as Olsen (2020), we categorized participant's debrief responses as follows: the participant provided (1) an 'I don't know' response or similar; (2) a description of the study that was completely unrelated to the topic; (3) a description of the study that was related to the topics investigated but with no sense of connection between them (i.e., mention of emotion or future or decision making without any connection). For options (4-6), participants provided a description of the study that was related to the topics investigated, and a sense of connection between them, with either (4) no reference to the prediction; (5) a reference to an incorrect prediction; (6) or a reference to the correct prediction. Table S4 shows the number of participants (+ \%) in each condition who fell into each category.

\section{Table S4}




\begin{tabular}{ccccccc}
\hline & \multicolumn{2}{c}{ Neutral } & \multicolumn{2}{c}{ Positive } & \multicolumn{2}{l}{ Negative } \\
\hline Response & $N$ & $\%$ & $N$ & $\%$ & $N$ & $\%$ \\
\hline 1 & 19 & 10.3 & 16 & 8.2 & 13 & 6.7 \\
2 & 22 & 11.9 & 18 & 9.3 & 17 & 8.8 \\
3 & 84 & 45.4 & 85 & 43.8 & 58 & 30.1 \\
4 & 55 & 29.7 & 63 & 32.5 & 89 & 46.1 \\
5 & 2 & 1.1 & 7 & 3.6 & 11 & 5.7 \\
6 & 3 & 1.6 & 5 & 2.6 & 5 & 2.6 \\
\hline
\end{tabular}

Fitting of hyperbolic discounting function in Bulley et al.'s data

As we used a different method to estimate hyperbolic discounting (based on Vincent, 2016), we compared the consistency of this approach with the values obtained for Bulley et al.'s (2019) data using their trial-wise data and associated scoring method (based on Kaplan et al., 2016). Reassuringly, we found a high degree of consistency between the two approaches [Neutral: $r(99)=.978$; Positive: $r(97)=.965$; Negative: $r(95)=.976 ;$ all $p$ 's<.001] 


\section{Supplementary References}

Bulley, A., Miloyan, B., Pepper, G. V., Gullo, M. J., Henry, J. D., \& Suddendorf, T. (2019). Cuing both positive and negative episodic foresight reduces delay discounting but does not affect risk-taking. Quarterly Journal of Experimental Psychology, 72(8), 1998-2017. https://doi.org/10.1177/1747021818819777

Kaplan, B. A., Amlung, M., Reed, D. D., Jarmolowicz, D. P., McKerchar, T. L., \& Lemley, S. M. (2016). Automating Scoring of Delay Discounting for the 21- and 27-Item Monetary Choice Questionnaires. The Behavior Analyst, 39(2), 293-304. https://doi.org/10.1007/s40614-016-0070-9

Olsen, R. (2020). The effect of episodic future thinking on delay discounting [Doctoral dissertation, Victoria University of Wellington].

Vincent, B. T. (2016). Hierarchical Bayesian estimation and hypothesis testing for delay discounting tasks. Behavior Research Methods, 48, 1608-1620.

doi:10.3758/s13428-015-0672-2 